\title{
The Foundations Framework for Developing and Report- ing New Models of Care for Multimorbidity
}

\author{
Jonathan Stokes, $\mathrm{PbD}, \mathrm{MPH}, \mathrm{BSc} \mathrm{c}^{1,2}$ \\ Mei-See Man, PbD, BSc ${ }^{3}$ \\ Bruce Gutbrie, MB BChir, MRCP, \\ MRCGP, MSc, $\mathrm{PbD}^{4}$ \\ Stewart W. Mercer, MBCbB, BSc, \\ $M S c, P b D, F R C G P^{5}$ \\ Chris Salisbury, $M B C b B, M S c, M D$, \\ FRCGP $P^{3}$
}

Peter Bower, $\mathrm{PbD}, \mathrm{BSc}^{6}$

'NIHR Greater Manchester Primary Care

Patient Safety Translational Research

Centre, Manchester Academic Health

Science Centre, University of Manchester,

Manchester, United Kingdom

${ }^{2}$ Centre for Health Economics, Manchester Academic Health Science Centre, School of Health Sciences, University of Manchester, Manchester, United Kingdom

${ }^{3}$ Centre for Academic Primary Care, School of Social and Community Medicine, University of Bristol, Bristol, United Kingdom

${ }^{4}$ Quality, Safety and Informatics Research Group, University of Dundee, Dundee,

United Kingdom

${ }^{5}$ General Practice and Primary Care, Institute for Health and Wellbeing, University

of Glasgow, Glasgow, Scotland, United Kingdom

${ }^{6}$ NIHR School for Primary Care Research, Centre for Primary Care, Manchester Academic Health Science Centre, School of Health Sciences, University of Manchester, Manchester, United Kingdom

\section{Sin}

MORE ONLINE

www.annfammed.org

Conflicts of interest: authors report none.

\section{CORRESPONDING AUTHOR}

Jonathan Stokes, PhD

Centre for Health Economics

Manchester Academic Health Science

Centre

School of Health Sciences

University of Manchester

Oxford Road

Manchester, UK M13 9PL

jonathan.m.stokes@manchester.ac.uk

\begin{abstract}
PURPOSE Multimorbidity challenges health systems globally. New models of care are urgently needed to better manage patients with multimorbidity; however, there is no agreed framework for designing and reporting models of care for multimorbidity and their evaluation.
\end{abstract}

METHODS Based on findings from a literature search to identify models of care for multimorbidity, we developed a framework to describe these models. We illustrate the application of the framework by identifying the focus and gaps in current models of care, and by describing the evolution of models over time.

RESULTS Our framework describes each model in terms of its theoretical basis and target population (the foundations of the model) and of the elements of care implemented to deliver the model. We categorized elements of care into 3 types: (1) clinical focus, (2) organization of care, (3) support for model delivery. Application of the framework identified a limited use of theory in model design and a strong focus on some patient groups (elderly, high users) more than others (younger patients, deprived populations). We found changes in elements with time, with a decrease in models implementing home care and an increase in models offering extended appointments.

CONCLUSIONS By encouragin greater clarity about the underpinning theory and target population, and by categorizing the wide range of potentially important elements of an intervention to improve care for patients with multimorbidity, the framework may be useful in designing and reporting models of care and help advance the currently limited evidence base.

Ann Fam Med 2017;15:570-577. https://doi.org/10.1370/afm.2150.

\section{INTRODUCTION}

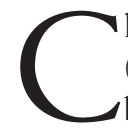
hronic disease is a global health priority, ${ }^{1,2}$ and multimorbidity (the coexistence of 2 or more chronic conditions in a patient) ${ }^{3}$ brings additional challenges. Patients with multimorbidity receive more fragmented care and have worse health outcomes, and health systems struggle to address their needs effectively. ${ }^{3} \mathrm{New}$ ways to deliver care are required to manage these patients' needs, especially in primary care, which often is responsible for their management. ${ }^{4-7}$

\section{Current Progress in Improving Care for Multimorbidity}

Large gaps exist in the evidence base concerning care for patients with multimorbidity. A recent Cochrane review found only 18 trials evaluating models of care. ${ }^{8}$ These models involved 2 broad strategies: reorganization of care delivery through enhanced multidisciplinary teamwork, and patient-oriented education or self-management. The review found limited evidence that the models examined were effective.

A major problem is the lack of consensus over the description of models of care for multimorbidity. If science is to drive clinical innovation, we need to build the evidence base through ongoing evaluation and review. That process is hampered, however, by incomplete descriptions of models 
in publications. ${ }^{9}$ Without complete, accurate descriptions, researchers cannot replicate studies or identify 'active ingredients.'.10

Developing a comprehensive framework for designing and reporting models for multimorbidity would provide a common understanding for researchers and clinicians, enable better description of existing and new models, and allow more effective analyses of what works for whom. This information is likely to be critical, given the broad range of approaches and patient populations included under the multimorbidity label. In developing our framework, we adopted Davidson et al's definition of a model of care as follows:

An overarching design for the provision of a particular type of health care service that is shaped by a theoretical basis, evidence-based practice and defined standards. It consists of defined core elements and principles and has a framework that provides the structure for the implementation and subsequent evaluation of care. ${ }^{11}$

We had the following aims: (1) to identify models of care relevant to the management of multimorbidity, (2) to analyze models in terms of their theoretical basis and core elements and develop a framework for the description of models of care, and (3) to illustrate the utility of the framework through applying it to explore how models have changed with time.

\section{METHODS}

\section{Identification of Models}

We carried out a large scoping review ${ }^{12}$ of models of primary care for chronic conditions and multimorbidity. We searched 3 bibliographic databases (MEDLINE, EMBASE, and Cochrane CENTRAL) with blocks of terms for multimorbidity/chronic conditions, AND primary care, AND models/frameworks/interventions (see the Supplemental Appendix at http:// www.AnnFamMed.org/content/15/6/570/suppl/DC1/ for search and model selection details). We supplemented our search with our knowledge of any additional models that fit our criteria. We did not exclude any model based on study type.

\section{Analysis and Framework Development}

We recorded the underlying theoretical basis of the model of care and extracted elements in each published article. We categorized these elements and formulated a glossary (see Supplemental Appendix). We also extracted details of the patient populations relevant to the model and provided a short description. We developed the framework iteratively as the data were extracted, with the authors reflecting through group discussion on the elements, the structure of those ele- ments, and common groupings of elements. Drawing from these discussions and the authors' own experience of multimorbidity and health systems research, we proposed a framework.

\section{Application of the Framework}

We used the framework to summarize the content of current models of care for multimorbidity, highlighting key issues arising from application of our framework to the models. To further illustrate use of the framework, we explored the evolution of models of care over time, comparing components of more recent models from 2010 onward with those of earlier models (giving an approximately equal number of models in each period) to illustrate changes over time. We used $\chi^{2}$ tests to compare the frequency with which elements were included before or after 2010 .

\section{RESULTS}

\section{Identification of Models}

The database search resulted in 15,880 titles. After screening and the addition of other relevant models known to the team, we included 39 different models of care (described in 68 articles). The Supplemental Appendix gives a brief description of each model (Table A1) and their elements (Table A2).

\section{Analysis and Framework Development}

Figure 1 illustrates the framework that arose from our analysis of the models and discussion of common groupings and descriptive properties. The foundations of the framework are the theoretical basis of the model and the defined patient population. We categorized the elements of the model into 3 areas: (1) clinical focus, (2) organization of care delivery, (3) support for model delivery.

\section{Foundations}

Theoretical Basis

The first foundation is the theoretical basis of the model. Models of care are designed generally to

Figure 1. The foundations framework.

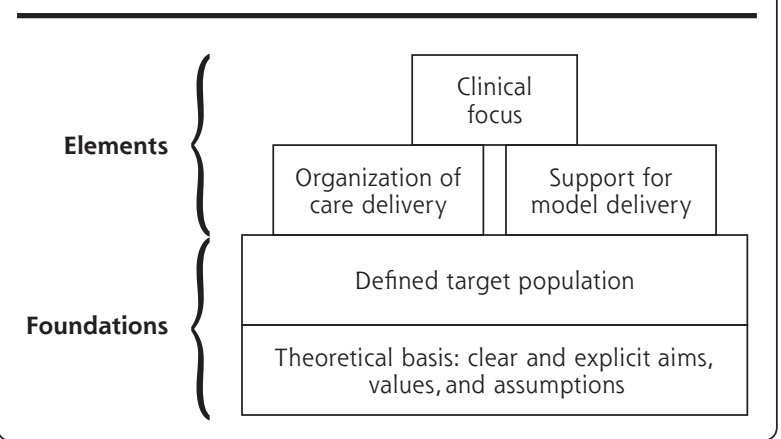


achieve a specific goal. For example, they might be designed to improve quality of care and reduce treatment burden for a group of patients with multimorbidity who previously had to attend multiple visits, or they might be designed to address a system's financial pressures. Models of care are therefore built (at least implicitly) on assumptions of how these goals can be achieved, which are sometimes outlined explicitly by reference to a theoretical basis. A theoretical basis does not usually specify in great detail the specific elements used in the model. ${ }^{13}$

\section{Target Population}

The second foundation is the defined target population. Despite similarities, chronic conditions vary widely in their manifestations and treatment indications. Similarly, people with multimorbidity are a heterogeneous population, with many subgroups and varying needs. A description of the population being targeted by a model is therefore essential.

\section{Elements}

Building on the theoretical basis and the target population, models have different elements that are implemented.

\section{Clinical Focus}

Many models of care change the clinical focus of care, and changes will be the most visible to the patients. Changes should align with the theoretical basis of the model (eg, shared decision making for patient-centered care), and might reflect the target population (eg, a focus on mental health).

\section{Organization of Care Delivery}

Models often change the organization of care delivery to allow or facilitate the necessary changes in clinical focus. For example, extended appointment times may be needed for shared decision making, or health coaches might be better suited than physicians to help the patients' self-manage. Specialist staff may be co-located in primary care clinics to prevent multiple visits and unnecessary patient burden.

\section{Support for Model Delivery}

Adapting the clinical focus and organization of care delivery is likely to require support for model delivery, including changes to supporting infrastructure, such as adaptions to workforce, technology, or incentives. For example, funding and payment modifications might be necessary to change clinician behaviors (eg, incentivizing preventative behavior), to better integrate care between sectors, or to increase the quantity of care delivered in the primary care setting.

\section{Application of the Framework}

Application of our framework to existing models of care for multimorbidity has highlighted the following issues.

\section{Foundations}

\section{Theoretical Basis}

Of the 39 models identified, only 17 (44\%) explicitly named a theoretical basis, and 15 of those (88\%) cited the chronic care model (CCM). The CCM describes several basic principles (use of self-management support, clinical information systems, delivery system redesign, decision support, health care organization, and community resources). ${ }^{14}$ In practice, models did not include all principles of the CCM. For example, few examined clinical information systems. Other theoretical bases included the patient-centered care model ${ }_{1}^{15}$ and the home-based palliative care programme model. ${ }^{16}$

\section{Target Populations}

Most models $(67 \%, \mathrm{n}=26)$ targeted only those at the highest risk of adverse events (most commonly hospitalizations) or the elderly. Only 1 model focused on those living in socioeconomically deprived areas. Only 2 models aimed to adapt care for their entire patient population (ie, taking a population health management approach through a patient-centered medical home) by expanding the target group to include more prevention-oriented clinical elements.

\section{Elements}

Figure 2 summarizes the elements identified in the current models of care. Below we describe elements in the 3 categories of clinical focus, organization of care delivery, and support for model delivery. Almost all models $(97 \%, \mathrm{n}=38)$ reported changes across all 3 categories, but with wide variation in specific elements deployed (see Supplemental Appendix Table A2).

\section{Clinical Focus}

In terms of clinical focus, most models included self-management support (87\%), a biopsychosocial approach (79\%), a focus on prevention $(74 \%)$, and attention to polypharmacy (72\%). About one-half of the models included emphasis on shared decision making $(56 \%)$, mental health $(54 \%)$, or a guidelineor protocol-based approach $(46 \%)$. We found little direct mention of treatment burden or quality of life (although these are perhaps implied in other categories, such as polypharmacy).

\section{Organization of Care Delivery}

We identified 13 elements for changing organization of care delivery. Most models included case management (90\%), integration with social and community care 


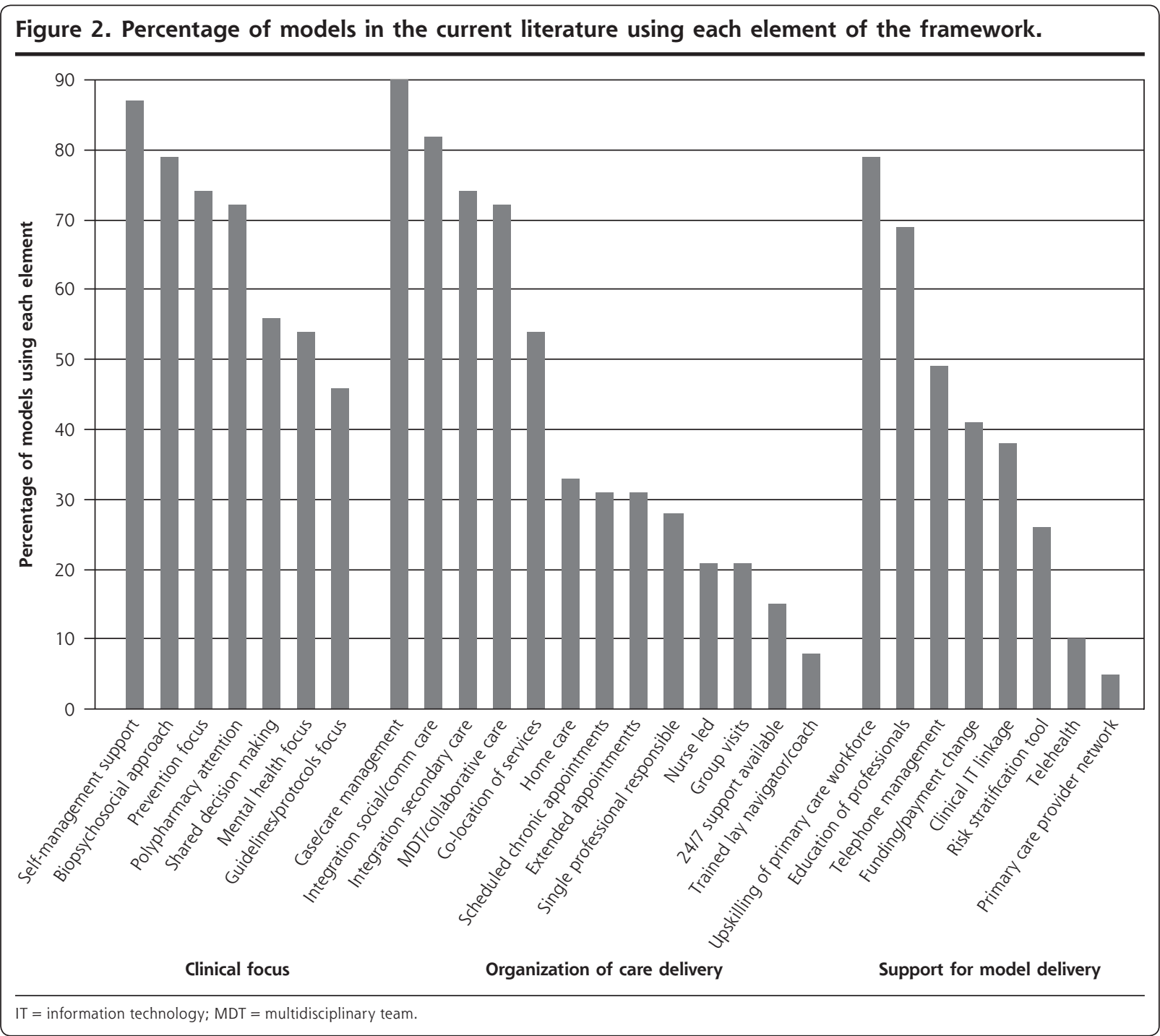

services $(82 \%)$ or secondary care $(74 \%)$, and a multidisciplinary approach $(72 \%)$. Slightly more than onehalf of the models had elements of home care (54\%). Less frequently included organizational changes were co-location of services (33\%), scheduled chronic disease appointments (31\%), extended appointment times (31\%), group visits $(21 \%)$, or $24 / 7$ support $(15 \%)$.

\section{Support for Model Delivery}

We identified 8 elements supporting model delivery, most commonly including up-skilling of the primary care workforce (introducing new roles to primary care, $79 \%)$, and education of professionals in chronic disease management and new ways of working (69\%). Nearly one-half included some telephone support for patients (49\%). Less frequently used elements were funding and payment changes (including incentives or changes to reimbursement mechanisms, $41 \%$ ), technology (such as clinical information technology linkage between services, $38 \%$ ), risk stratification tools $(26 \%)$, or telehealth (remote care and monitoring separate from telephone management, 10\%).

\section{Change in Models of Care for Multimorbidity With Time}

Application of our framework showed the following changes with time (Figure 3).

The only statistically significant changes identified were in terms of organization of care delivery, with a decrease in models implementing home care $(-45 \%)$ and an increase in models offering extended appointments $(+37 \%)$. There were other trends $(10 \%$ or more change in either direction) tending to favor expansion of primary care services in a single location (eg, increased co-location of social care services and extended chronic disease appointments), rather than 
coordination across multiple providers (eg, decreased care planning and integration with other social and community care services).

Two elements have emerged that did not occur before 2010 (trained lay navigators and coaches, and primary care provider networks). The proportion of models utilizing each element over time are shown in the Supplemental Appendix Table A3.

\section{DISCUSSION}

We have described a framework for reporting and designing models of care for multimorbidity. The framework identifies 2 foundations, the theoretical basis, and defined target population, and 3 categories of care elements to implement the model in practice, including (1) clinical focus (2) organization of care delivery, and (3) support for model delivery. We have provided a list of elements that have been used to date. We have used the framework to identify the focus and gaps in current models, as well as developments over time.

\section{Limitations of the Study}

The search strategy used to develop the framework was restricted to published models, but was inclusive regardless of study type. It is likely, however, that individual elements deployed will continue to change with

\section{Figure 3. Model development over time.}

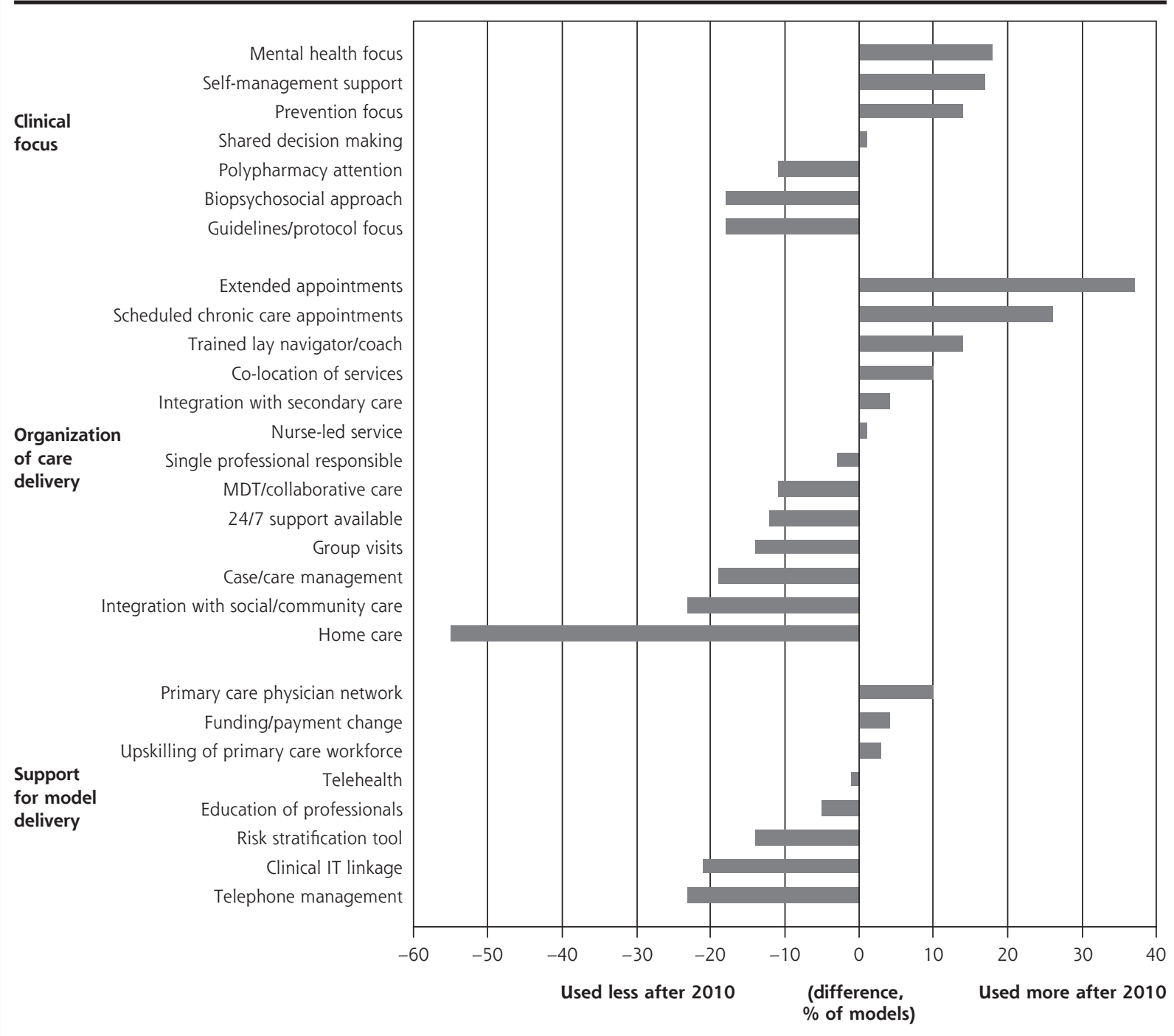

IT = information technology; MDT = multidisciplinary team.

Note: Difference $=$ the percentage of models described in 2010 or later implementing the element less the percentage of models described before 2010 implementing the element. 
time, although we expect that the broad framework will endure even when new elements are added. We would highlight that the detail required to develop a usable framework is not the same as that required for replication of models in practice. We would therefore recommend that this framework be considered a minimum rather than maximum level of detail for model description in future research (supplementary to existing research reporting checklists). ${ }^{9}$ Our framework is not designed to be prescriptive about elements to be used in new models, as the current evidence about 'active ingredients' is so limited. The design and implementation of models will also need to be sensitive to context. ${ }^{17}$ Use of our framework could allow assessment of the contribution of different components in the future, however, through meta-regression or detailed qualitative process evaluations. ${ }^{10,18}$

\section{Interpretation in the Context of the Wider Literature}

The multimorbidity Cochrane review ${ }^{8}$ and other published reviews $\mathrm{s}^{19,20}$ have proposed preliminary classifications of models for multimorbidity, but none has been comprehensive or included new approaches that have not been subject to published trials. Our framework builds on and extends the categorization used in the recent Cochrane review on effectiveness of multimorbidity models. The National Institute for Health and Care Excellence (NICE) recently issued clinical guidelines for multimorbidity, ${ }^{21}$ and our framework complements that by outlining some of the wider changes necessary to support the concept of tailored care in the health system.

\section{Implications of the Framework for Policy and Practice}

The theoretical basis of models highlights the assumptions made by the designers. For example, designers may vary in the extent to which they ascribe responsibility for health to individuals or to wider social context. At present, the basis of models appears mostly homogenous, with the most frequent being the CCM. The CCM was initially designed and implemented for single disease-management programs, ${ }^{13,22}$ and there is an argument that this approach is not sensitive to the needs of multimorbidity (eg, with an emphasis on disease-specific guideline-based decision support). ${ }^{23}$ There are fewer models based on more recent developments, such as the "minimally disruptive medicine" model. ${ }^{24}$ Changes in theoretical basis may have important implications for model design. For example, models based on minimally disruptive medicine may affect the clinical focus on selfmanagement, as increased emphasis on self-management may increase the burden of care for a patient.
In terms of population, our mapping of models to the framework determined that most models have focused on a select group, such as older people or those at high risk (most commonly of hospital admission). The highest risk patients make up an extremely small proportion of the total population and of those with multimorbidity, and they may already be past the point of care having major impact on disease course..$^{25-28}$ Although older people have more conditions on average, in absolute terms fewer people with multimorbidity are aged 65 years and older, ${ }^{29}$ so it is also important that relevant models are designed for younger and lower risk patients. Although these currently less-targeted groups do not (yet) account for most secondary care admissions and the total cost of care, it may be possible to alleviate their health care demand in the future (eg, through compression of multimorbidity into a smaller period at the end of life). ${ }^{30}$ Furthermore, few interventions targeted the most socioeconomically deprived populations, where multimorbidity is known to be more common, occur at an earlier age, and be more likely related to poor mental health. ${ }^{29,31}$

Our analysis shows several elements of care that are in need of increased attention. Notably, a mental health focus is lacking from nearly one-half of all models we included (although a focus on mental health has increased with time). Multimorbid patients' with mental health issues are at increased risk for patient safety incidents in primary care, ${ }^{31}$ and depression is particularly important in modifying multimorbidity management and outcomes. ${ }^{32}$ Few of the models directly focus on treatment burden, which is increasingly recognized as an important phenomenon for people with multimorbidity.

Conversely, some of the elements included most commonly do not have a strong evidence base or may be of questionable value in multimorbidity. The emphasis on self-management and prevention may be inappropriate, because high levels of morbidity are associated with barriers to self-management, ${ }^{33}$ and the NICE guidelines found no evidence to support recommending such activities in patients with multimorbidities. ${ }^{21}$ The potential for prevention in an elderly high-risk population is debatable. ${ }^{26}$ Similarly the evidence for case management suggests that patient satisfaction can be improved, but cost and self-assessed health are not significantly affected ${ }_{1}^{34}$ particularly for the most complex patients and even when conducted by a multidisciplinary team. ${ }^{35}$ Finally, although several models were based on reorganization of services using telehealth, evidence suggests this approach may not be cost-effective for managing chronic conditions. ${ }^{36}$

Health systems have only recently begun to implement new models of care for multimorbidity, with 
limited evidence of success. Careful design, implementation, and reporting can assist in the development of the evidence base in this important area. We hope our framework can encourage more standardized reporting and research on the theoretical basis and target population for interventions, as well as the contribution of different elements (including interactions between them) needed to provide cost-effective care and support redesign of health systems for those who use them most. ${ }^{37}$

To read or post commentaries in response to this article, see it online at http://www.AnnFamMed.org/content/15/6/570.

Submitted December 7, 2016; submitted, revised, May 9, 2017; accepted June 15, 2017.

Key words: framework; chronic disease; multimorbidity; models of care; primary care; health systems

Funding support: All authors except Dr Stokes were funded by the National Institute for Health Research (NIHR) Health Services and Delivery Research (project No 12/130/15). Dr Stokes was funded by the NIHR Greater Manchester Primary Care Patient Safety Translational Research Centre (NIHR GM PSTRC).

Disclaimer: The views and opinions expressed therein are those of the authors and do not necessarily reflect those of the HTA program, NIHR, National Health Service, or the Department of Health. The funder played no role in the study design, in the collection, analysis or interpretation of the data, in the writing of the paper, or in the decision to submit the paper for publication. All authors were independent from the funders.

Author contributions: J.S., M-S. M., B.G., S.W.M., C.S., and P.B. conceived the study. J.S. led its development and execution. J.S. and M-S.M. conducted the initial study screening, selection, and extraction. Thereafter, J.S., M-S.M., B.G., S.W.M., C.S., and P.B. contributed to various aspects of the empirical work, analysis and presentation. J.S., M.S.M., B.G., S.W.M., C.S., and P.B. drafted the manuscript and all authors reviewed it and approved the final version. J.S. is the guarantor.

Supplementary materials: Available at http://www.AnnFamMed. org/content/15/6/570/suppl/DC1/.

\section{References}

1. Omran AR. The epidemiologic transition. A theory of the epidemiology of population change. Milbank Mem Fund Q. 1971;49(4): 509-538.

2. Boutayeb A. The double burden of communicable and noncommunicable diseases in developing countries. Trans $R$ Soc Trop Med Hyg. 2006;100(3):191-199.

3. Boyd CM, Fortin M. Future of multimorbidity research: How should understanding of multimorbidity inform health system design? Public Health Rev. 2011;33(2):451-474.

4. Nolte E, McKee M. Caring for people with chronic conditions: a health system perspective. 2008. http://www.euro.who.int/__data/ assets/pdf_file/0006/96468/E91878.pdf. Accessed Jan 10, 2013.

5. World Health Organization. WHO global strategy on peoplecentred and integrated health services: interim report. 2015. http://apps.who.int/iris/bitstream/10665/155002/1/WHO_HIS_ SDS_2015.6_eng.pdf. Accessed Jan 2, 2016.
6. England NHS. Five Year Forward View. 2014. https://www.england. nhs.uk/wp-content/uploads/2014/10/5yfv-web.pdf. Accessed Jan 11, 2015.

7. Bayliss EA. Simplifying care for complex patients. Ann Fam Med. 2012;10(1):3-5.

8. Smith SM, Wallace E, O'Dowd T, Fortin M. Interventions for improving outcomes in patients with multimorbidity in primary care and community settings. Cochrane Database Syst Rev. 2016;3:CD006560.

9. Hoffmann TC, Glasziou PP, Boutron I, et al. Better reporting of interventions: template for intervention description and replication (TIDieR) checklist and guide. BMJ. 2014;348:g1687.

10. Moore GF, Audrey S, Barker M, et al. Process evaluation of complex interventions: Medical Research Council guidance. BMJ. 2015;350: h1258.

11. Davidson P, Halcomb E, Hickman L, et al. Beyond the rhetoric: what do we mean by a 'model of care'? Aust J Adv Nurs. 2006; 23(3):47-55.

12. Arksey H, O'Malley L. Scoping studies: towards a methodological framework. Int J Soc Res Methodol. 2005;8(1):19-32.

13. Bodenheimer T, Wagner EH, Grumbach K. Improving primary care for patients with chronic illness. JAMA. 2002;288(14):1775-1779.

14. Wagner EH. Chronic disease management: what will it take to improve care for chronic illness? Eff Clin Pract. 1998;1(1):2-4.

15. Man M-S, Chaplin K, Mann C, et al. Improving the management of multimorbidity in general practice: protocol of a cluster randomised controlled trial (The 3D Study). BMJ Open. 2016;6(4):e011261.

16. Levine S, Steinman BA, Attaway K, Jung T, Enguidanos S. Home care program for patients at high risk of hospitalization. Am 」 Manag Care. 2012;18(8):e269-e276.

17. Craig $P$, Dieppe $P$, Macintyre $S$, et al. Developing and evaluating complex interventions: the new Medical Research Council guidance. BMJ. 2008;337:a1655.

18. Bower P, Gilbody S, Richards D, Fletcher J, Sutton A. Collaborative care for depression in primary care. Making sense of a complex intervention: systematic review and meta-regression. Br J Psychiatry. 2006;189:484-493.

19. Bleich SN, Sherrod C, Chiang A, et al. Systematic review of programs treating high-need and high-cost people with multiple chronic diseases or disabilities in the United States, 2008-2014 [published correction appears in Prev Chronic Dis. 2016;13:E31]. Prev Chronic Dis. 2015;12:E197.

20. Bienkowska-Gibbs T, King S, Saunders C, et al. New Organisational Models of Primary Care to Meet the Future Needs of the NHS: A Brief Overview Of Recent Reports. Cambridge, England: RAND Europe; 2015.

21. NICE. Multimorbidity: clinical assessment and management NICE guideline. 2016. https://www.nice.org.uk/guidance/ ng56/resources/multimorbidity-clinical-assessment-andmanagement-1837516654789. Accessed Sep 22, 2016.

22. Bodenheimer T, Wagner EH, Grumbach K. Improving primary care for patients with chronic illness: the chronic care model, Part 2. JAMA. 2002;288(15):1909-1914.

23. Boyd CM, Darer J, Boult C, Fried LP, Boult L, Wu AW. Clinical practice guidelines and quality of care for older patients with multiple comorbid diseases: implications for pay for performance. JAMA. 2005;294(6):716-724.

24. May C, Montori VM, Mair FS. We need minimally disruptive medicine. BMJ. 2009; 339:b2803. http://www.bmj.com/content/339/bmj. b2803.

25. Wallace E, Salisbury C, Guthrie B, Lewis C, Fahey T, Smith SM. Managing patients with multimorbidity in primary care. BMJ. 2015;350: h176. 
26. Wallace $E$, Smith SM, Fahey T, Roland M. Reducing emergency admissions through community based interventions. BMJ. 2016; 352:h6817.

27. Roland M, Abel G. Reducing emergency admissions: are we on the right track? BMJ. 2012;345:e6017.

28. McWilliams JM, Schwartz AL. Focusing on high-cost patients - the key to addressing high costs?. N Engl J Med. 2017;376(9):807-809.

29. Barnett K, Mercer SW, Norbury M, Watt G, Wyke S, Guthrie B. Epidemiology of multimorbidity and implications for health care, research, and medical education: a cross-sectional study. Lancet. 2012;380(9836):37-43.

30. Fries JF. Aging, natural death, and the compression of morbidity. N Engl J Med. 1980;303(3):130-135.

31. Panagioti M, Stokes J, Esmail A, et al. Multimorbidity and patient safety incidents in primary care: a systematic review and metaanalysis. PLoS One. 2015;10(8):e0135947.

32. Moussavi S, Chatterji S, Verdes E, Tandon A, Patel V, Ustun B. Depression, chronic diseases, and decrements in health: results from the World Health Surveys. Lancet. 2007;370(9590):851-858.
33. Bayliss EA, Ellis JL, Steiner JF. Barriers to self-management and quality-of-life outcomes in seniors with multimorbidities. Ann Fam Med. 2007;5(5):395-402.

34. Stokes J, Panagioti M, Alam R, Checkland K, Cheraghi-Sohi S, Bower P. Effectiveness of case management for 'at risk' patients in primary care: a systematic review and meta-analysis. PLoS One. 2015;10(7):e0132340.

35. Stokes J, Kristensen SR, Checkland K, Bower P. Effectiveness of multidisciplinary team case management: difference-in-differences analysis. BMJ Open. 2016;6(4):e010468.

36. Henderson C, Knapp M, Fernández J-L, et al. Cost effectiveness of telehealth for patients with long term conditions (Whole Systems Demonstrator telehealth questionnaire study): nested economic evaluation in a pragmatic, cluster randomised controlled trial. BMJ. 2013;346.

37. Salisbury C. Multimorbidity: redesigning health care for people who use it. Lancet. 2012;380(9836):7-9. 